\title{
Pro PHP XML and Web Services
}

Robert Richards 


\section{Pro PHP XML and Web Services \\ Copyright $\odot 2006$ by Robert Richards}

All rights reserved. No part of this work may be reproduced or transmitted in any form or by any means, electronic or mechanical, including photocopying, recording, or by any information storage or retrieval system, without the prior written permission of the copyright owner and the publisher.

Library of Congress Cataloging-in-Publication data is available upon request.

Trademarked names may appear in this book. Rather than use a trademark symbol with every occurrence of a trademarked name, we use the names only in an editorial fashion and to the benefit of the trademark owner, with no intention of infringement of the trademark.

Lead Editor: Matt Wade

Technical Reviewers: Christian Stocker, Adam Trachtenberg

Editorial Board: Steve Anglin, Dan Appleman, Ewan Buckingham, Gary Cornell, Jason Gilmore, Jonathan Hassell, James Huddleston, Chris Mills, Matthew Moodie, Dominic Shakeshaft, Jim Sumser, Matt Wade

Project Manager: Kylie Johnston

Copy Edit Manager: Nicole LeClerc

Copy Editor: Kim Wimpsett

Assistant Production Director: Kari Brooks-Copony

Production Editor: Kelly Gunther

Artist: Kinetic Publishing Services, LLC

Cover Designer: Kurt Krames

Manufacturing Director: Tom Debolski

Distributed to the book trade worldwide by Springer-Verlag New York, Inc., 233 Spring Street, 6th Floor, New York, NY 10013. Phone 1-800-SPRINGER, fax 201-348-4505, e-mail orders-ny@springer-sbm.com, or visit http://www. springeronline.com.

For information on translations, please contact Apress directly at 2560 Ninth Street, Suite 219, Berkeley, CA 94710. Phone 510-549-5930, fax 510-549-5939, e-mail info@apress.com, or visit http: / www. apress.com.

The information in this book is distributed on an "as is" basis, without warranty. Although every precaution has been taken in the preparation of this work, neither the author(s) nor Apress shall have any liability to any person or entity with respect to any loss or damage caused or alleged to be caused directly or indirectly by the information contained in this work.

The source code for this book is available to readers at http://wWw. apress. com in the Source Code section.

This book is dedicated to my wife and best friend, Julie. Thank you for your patience, support, and encouragement at the times I most needed it. 


\section{Contents}

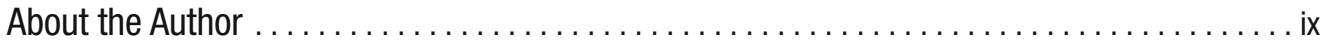

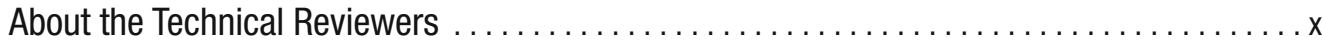

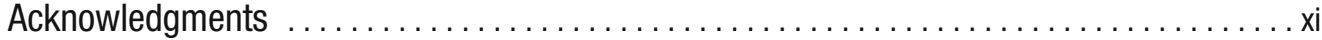

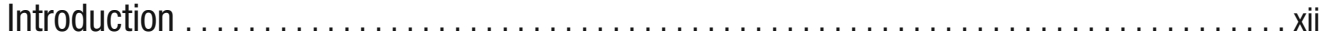

CHAPTER 1 Introduction to XML and Web Services $\ldots \ldots \ldots \ldots \ldots \ldots$

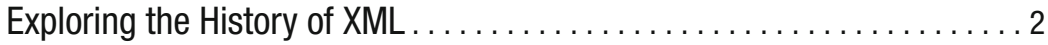

Using XML in the Real World ........................... 4

Introducing Service Oriented Architecture and Web Services .......... 9

Defining Common Terms and Acronyms ..................... 14

Conclusion ...................................... 14

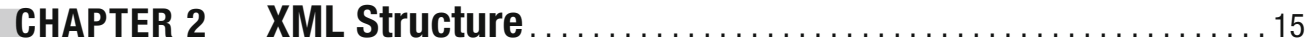

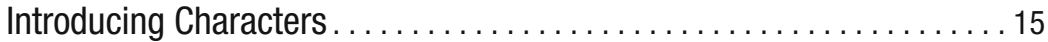

Understanding Basic Layout . . . . . . . . . . . . . . . . . . . . 18

Understanding Basic Syntax . . . . . . . . . . . . . . . . . . . . . 20

Using Namespaces . . . . . . . . . . . . . . . . . . . . . . . . . 29

Using IDs, IDREF/IDREFS, and xml:id . . . . . . . . . . . . . . . 36

Using xml:space and xml:lang $\ldots \ldots \ldots \ldots \ldots \ldots \ldots \ldots \ldots \ldots \ldots \ldots$

Understanding XML Base ............................. 42

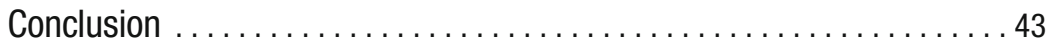

CHAPTER 3 Validation....................................... 45

Introducing Validation ............................. 45

Introducing Document Type Definitions. .................... 46

Using XML Schemas .................................. 71

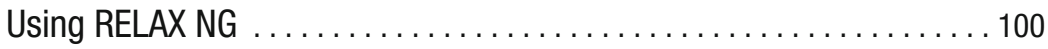

Conclusion ........................................ 121

CHAPTER 4 XPath, XPointer, XInclude, and the Future $\ldots \ldots \ldots \ldots 123$

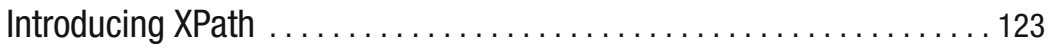

Introducing XPointer ................................ 146 


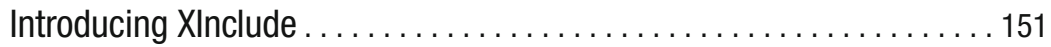

Examining the Future of XML . . . . . . . . . . . . . . . . . . . . . 157

Conclusion ....................................... 161

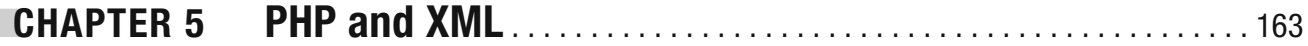

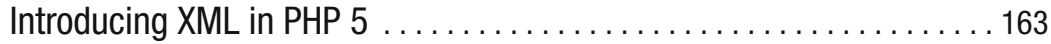

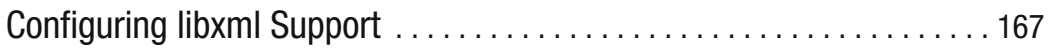

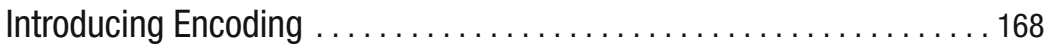

Figuring Out the libxml2 Version....................... 172

Introducing Parser Options ............................ 173

Introducing PHP Streams .............................. 174

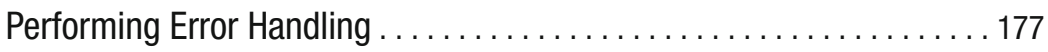

Conclusion ......................................... 179

CHAPTER 6 Document Object Model (DOM) ................... 181

Introducing the DOM .............................. 181

Using the DOM Extension. ............................ 188

Performing Validation ................................ 214

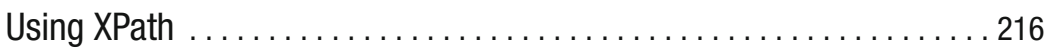

Extending Classes ................................... 219

Common Questions, Misconceptions, and Problems ............ 223

Migrating from domxml to the DOM Extension ................ 228

Seeing Some DOM Examples .......................... 230

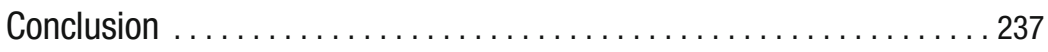

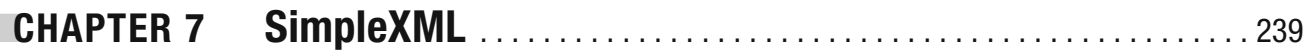

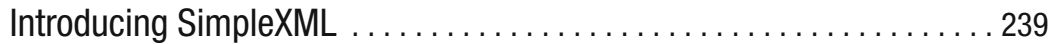

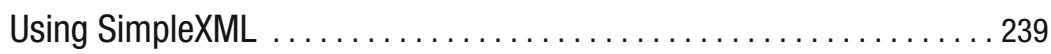

Using Namespaces in SimpleXML $\ldots \ldots \ldots \ldots \ldots \ldots \ldots \ldots \ldots \ldots \ldots$

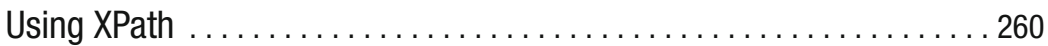

Seeing Some Examples in Action . . . . . . . . . . . . . . . . . . 262

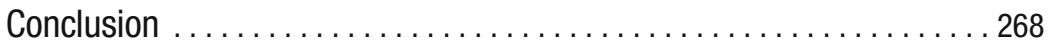

CHAPTER 8 Simple API for XML (SAX) ...................... 269

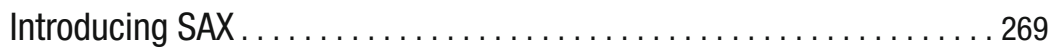

Using the $x m l$ Extension............................... 270

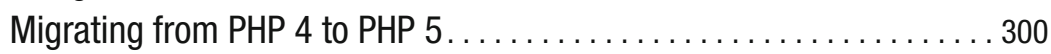


Seeing Some Examples in Action . . . . . . . . . . . . . . . . . 306

Conclusion ........................................... 310

CHAPTER $9 \quad$ XMLReader ........................................ 311

Introducing XMLReader .............................. 311

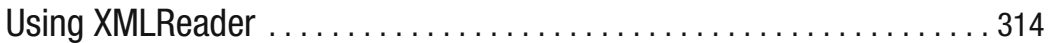

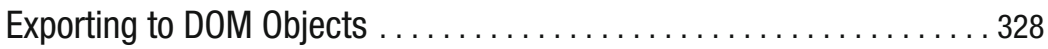

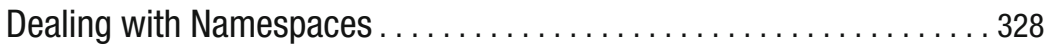

Performing Validation ................................. 333

Seeing Some Examples in Action . . . . . . . . . . . . . . . . . . . . . 335

Conclusion ........................................... 340

CHAPTER 10 Extensible Stylesheet Language Transformations

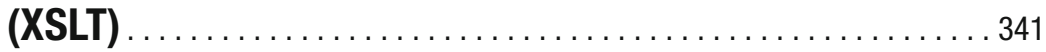

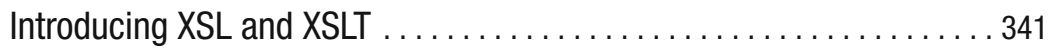

Introducing the XSL Extension. ......................... 387

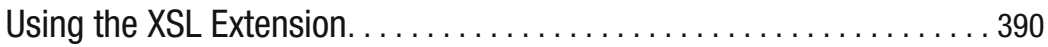

Using Parameters in XSL . . . . . . . . . . . . . . . . . . . . . . . . . . 393

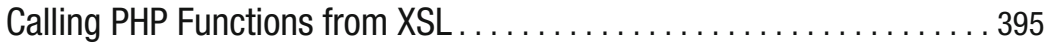

Seeing Some Examples in Action . . . . . . . . . . . . . . . . . . . . 399

Conclusion ......................................... 408

CHAPTER 11 Effective and Efficient Processing ................ 409

Looking at the Pros and Cons of Parsers .................... 409

Optimizing Parsing and Processing ....................... 426

Combining Technologies............................. 433

Conclusion ....................................... 439

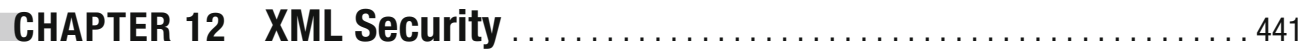

Introducing XML Security............................. 441

Introducing Basic Security . ........................... 442

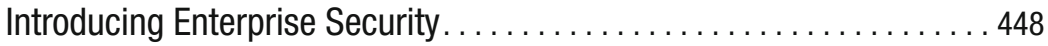

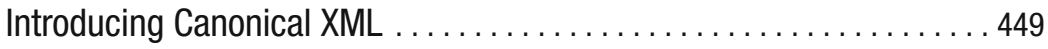

Introducing Exclusive XML Canonicalization. ................. 456

Introducing XML Signatures . . . . . . . . . . . . . . . . . . . . . . . 460

Introducing XML Encryption. ......................... 474

Conclusion ...................................... 489 


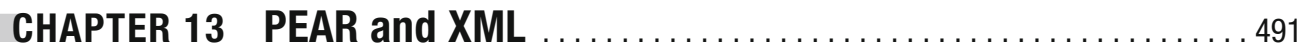

What Is PEAR? . . . . . . . . . . . . . . . . . . . . . . . 491

Using PEAR . . . . . . . . . . . . . . . . . . . . . . . . . 492

Using PEAR and XML Together ...................... 493

Conclusion ................................. 519

CHAPTER 14 Content Syndication: RSS and Atom ................ 521

Understanding the Evolution of RSS and Atom . . . . . . . . . . . 521

Introducing RSS 1.0: RDF Site Summary . . . . . . . . . . . . . 523

Introducing RSS 2.0: Really Simple Syndication . . . . . . . . . . . . . . 534

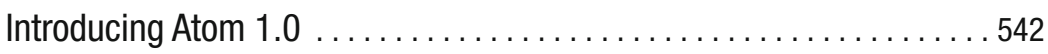

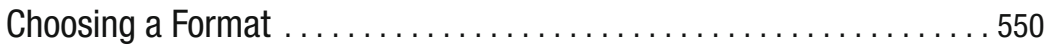

Seeing Some Examples in Action . . . . . . . . . . . . . . . . . . 551

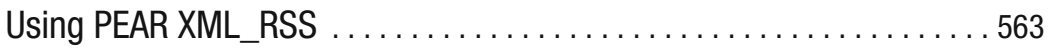

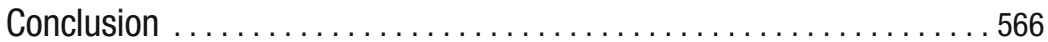

CHAPTER 15 Web Distributed Data Exchange (WDDX) ............. 567

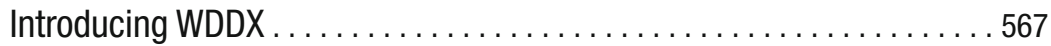

Understanding the Structure of WDDX . . . . . . . . . . . . . . . . . 569

Using WDDX . . . . . . . . . . . . . . . . . . . . . . . . . 576

Seeing Some Examples in Action . . . . . . . . . . . . . . . . . . . . 583

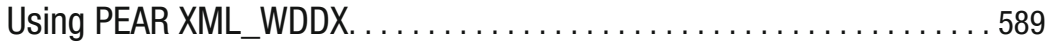

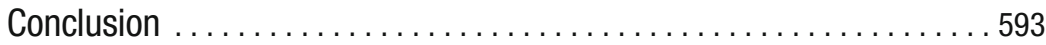

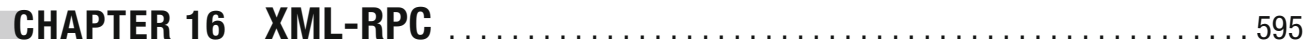

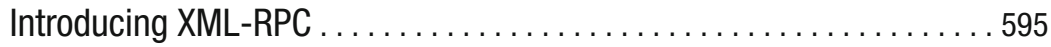

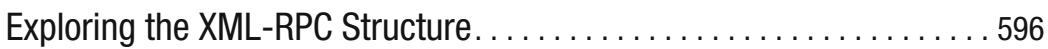

Using xmlrpc in PHP . . . . . . . . . . . . . . . . . . . . 608

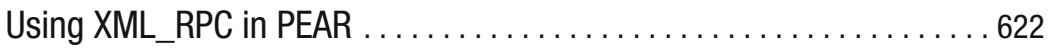

Seeing Some Examples in Action . . . . . . . . . . . . . . . . . . . . 629

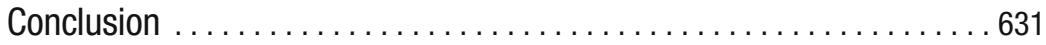

CHAPTER 17 Representational State Transfer (REST) . . . . . . . . . 633

Introducing REST . . . . . . . . . . . . . . . . . . . . . . 633

Introducing REST Web Services . . . . . . . . . . . . . . . . . 634

Creating a REST Web Service . . . . . . . . . . . . . . . . . . . . 639

Introducing the Yahoo Web Services $\ldots \ldots \ldots \ldots \ldots \ldots \ldots \ldots 6$ 
Introducing the Amazon Web Services. . . . . . . . . . . . . . 660

Conclusion . . . . . . . . . . . . . . . . . . . . . . . . . . . . . . . . . . . 672

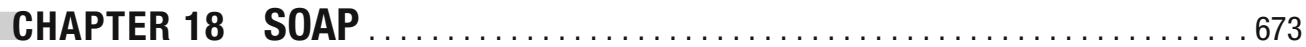

Introducing the Web Services Description Language (WSDL) . . . . . . 673

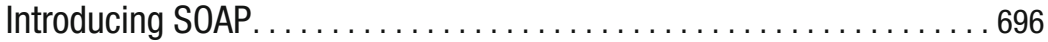

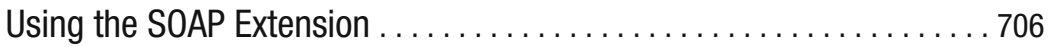

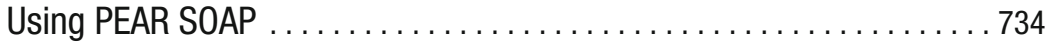

Seeing Some Examples in Action . . . . . . . . . . . . . . . . . . . 735

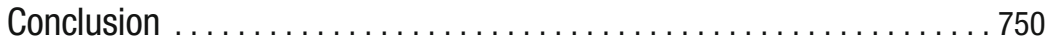

CHAPTER 19 Universal Description, Discovery, and

Integration (UDDI) ............................ 751

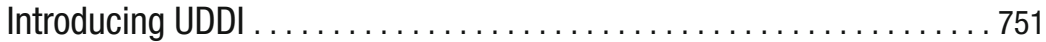

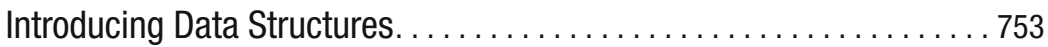

Introducing the SOAP API. . . . . . . . . . . . . . . . . . . . 764

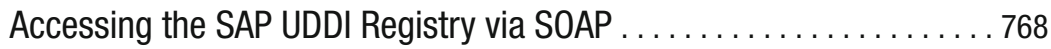

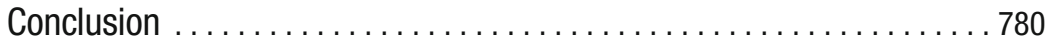

CHAPTER 20 PEAR and Web Services ....................... 781

Using Services_Amazon. ........................ 781

Using Services_Delicious.......................... 785

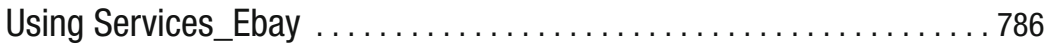

Using Services_Google........................... 786

Using Services_Technorati . . . . . . . . . . . . . . . . . . . . . 789

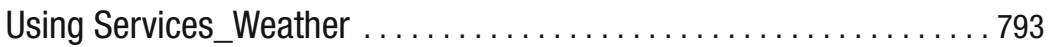

Using Services_Webservice . . . . . . . . . . . . . . . . . . . . 797

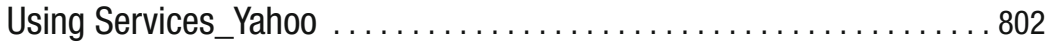

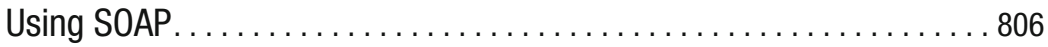

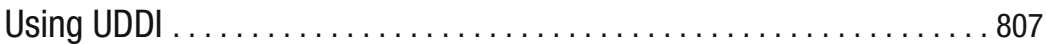

Using XML_RPC $\ldots \ldots \ldots \ldots \ldots \ldots \ldots \ldots \ldots \ldots \ldots \ldots \ldots \ldots$

Conclusion . . . . . . . . . . . . . . . . . . . . . . . . . 809

CHAPTER 21 0ther XML Technologies and Extensions .............811

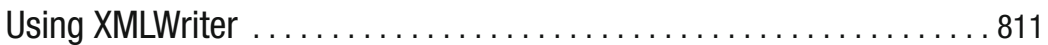

Using SD0 XML Data Access Service . . . . . . . . . . . . . 820

Introducing Asynchronous JavaScript Technology and XML (Ajax) . . . . 826 


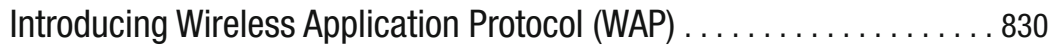

Conclusion ................................. 838

APPENDIX A XML Schema Built-in Data Types Reference ........... 839

Type Definition $\ldots \ldots \ldots \ldots \ldots \ldots \ldots \ldots \ldots \ldots \ldots \ldots \ldots \ldots . \ldots \ldots$

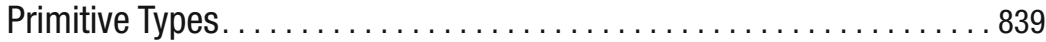

Derived Types. . . . . . . . . . . . . . . . . . . . . . 841

APPENDIX B Extension APIs ................................. 845

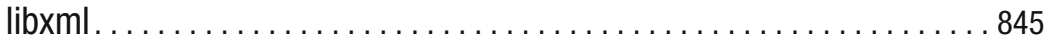

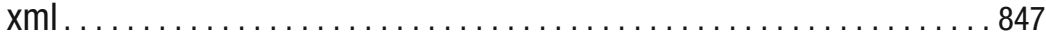

XMLReader .................................... 849

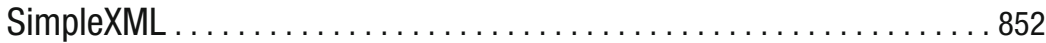

DOM. . . . . . . . . . . . . . . . . . . . . . . . . . . . 854

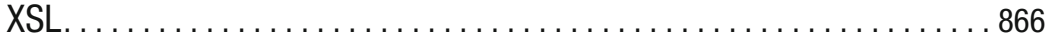

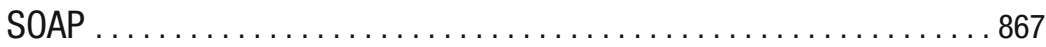

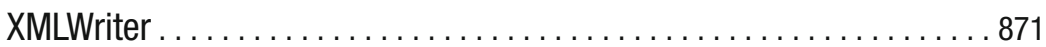

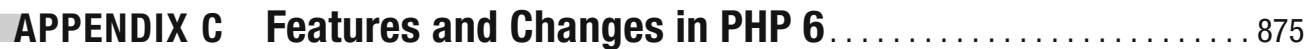

xml Extension. . . . . . . . . . . . . . . . . . . . . . . . 875

XMLReader Extension. . . . . . . . . . . . . . . . . . . . . . . . 876

SimpleXML Extension . . . . . . . . . . . . . . . . . . . . . . 879

D0M Extension. . . . . . . . . . . . . . . . . . . . . . . . . . . 883 


\section{About the Author}

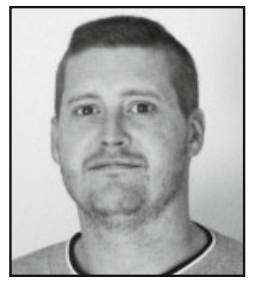

ROB RICHARDS, currently an independent contractor, has worked in various fields including medical information, telecommunications, media, and e-learning. Having been exposed to XML since its inception, he has used the technology for various projects throughout his career; his most extensive work with XML was within the e-learning space. He helped create a proprietary XML-based application server that used XML for data publishing, defining application business logic, and data querying. $\mathrm{He}$ was also the lead engineer for the company's involvement in the Shareable Content Object Reference Model (SCORM), which is used for Web-based learning and was established by the Department of Defense through its Advanced Distributed Learning (ADL) initiative.

After becoming the latest casualty of the dot-com implosion in 2001, Rob got his first taste of PHP and began contributing code to the domxml extension in 2002. Since then, he has become one of the authors of the DOM extension for PHP 5; he also contributes to the other XML-based extensions and authored the XMLReader and XMLWriter extensions. Also, on occasion, he contributes bug fixes to the libxml2 project for bugs found during the development of these extensions. 


\section{About the Technical Reviewers}

CHRISTIAN STOCKER is one of the developers of numerous XML extensions in PHP and has been involved in developing PHP since version 4.1.

In addition, he has been a speaker for many international conferences (ApacheCon, PHP Conference, and OSCOM) and actively takes part in the open source community. He's also the author of the German book PHP de Luxe, recently republished in its second edition.

In his day job, he is the $\mathrm{CEO}$ of Bitflux $\mathrm{GmbH}$, a Web development company specializing in XML/XSLT, PHP, and Ajax and based in Zurich, Switzerland.

ADAM TRACHTENBERG is the senior manager of platform evangelism at eBay, where he preaches the gospel of the eBay platform to developers and businesspeople around the globe. Before eBay, Adam cofounded and served as vice president for development at two companies, Student.com and TVGrid.com. At both firms, he led the front- and middle-end Web site design and development. Adam began using PHP in 1997; he is the author of Upgrading to PHP 5 (O'Reilly, 2004) and the coauthor of PHP Cookbook (O'Reilly, 2002). He lives in San Francisco, blogs at http: //www.trachtenberg.com, and has a bachelor's degree and a master's degree from Columbia University. 


\section{Acknowledgments}

would like to thank both Christian Stocker and Adam Trachtenberg for taking time out of their busy schedules to perform technical reviews of this book. The comments and feedback were invaluable to its completion. I also cannot forget to mention all the contributions from all the PHP developers who wrote and contributed to the various XML extensions in PHP 5, as well as Daniel Veillard and the maintainers of the libxml2 and libxslt libraries. Without all the hard work of these people, it is uncertain what the state of XML would be in PHP. I would also like to thank Matt Wade, Kylie Johnston, Kim Wimpsett, and the rest of the staff at Apress for making this book possible.

On a more personal note, a special thanks goes out to my family: my parents, Brian and Lillian; my wife, Julie; and her parents, Tony and Val. You all encouraged me during the entire book process and kept me going when things got difficult. 


\section{Introduction}

$\mathbf{X}$

ML and its associated technologies have been around for many years. Although some support has been available, it has not always been easy to work with XML using PHP. This all changed with the release of PHP 5. The inclusion of a variety of XML processors provides a developer with an arsenal of tools to tackle virtually any type of challenge involving XML. PHP 5 also went the extra step with the creation of the SOAP extension, providing native SOAP client and server support and allowing a developer to quickly and easily consume or create Web services.

With all these tools now available, PHP has become a more viable solution to implement applications that involve XML and Web services. The problem is that it is often difficult for a developer to understand how to begin using any of these tools. Not only do you need to understand the APIs of these extensions, but you also need to know which extension to use. On top of all this, you also need to understand the specifications for the different XML technologies.

This book takes a different approach than most on this subject. Pro PHP XML and Web Services provides an in-depth and comprehensive look at not only the tools available with PHP but also the specifications for a variety of XML-based tools. An understanding of the specifications is often critical when developing an XML-based application. After all, a tool is only good as your understanding of what you can do with it. However, the problem with the specifications is that they tend to be overly complex. For this reason, I will explain them in easy-to-understand language and include complete examples. Specifically, I take the concepts from the technical specifications and show how to adapt them to real-world use in PHP by covering the APIs and areas of functionality and showing examples of their usage.

Regardless of whether you are a novice or a more advanced developer in the area of XML, the material presented in this book will get you developing XML-based applications in PHP faster, and it will demonstrate how to maximize your usage of the XML tools now supported in PHP.

\section{Who This Book Is For}

This book is for developers of all skill levels looking to use XML in PHP. I explain the XML technologies and PHP extensions in easy-to-understand terms and examples. This will allow developers new to XML or Web services to start coding right away instead of spending countless hours deciphering the often-cryptic specifications and documentation. Developers already proficient in XML will find techniques and information about interoperability, optimization, and undocumented features of some of the XML-based extensions in order to maximize the effectiveness of an XML or Web service-based application they may be writing. 


\section{How This Book Is Structured}

For you to get the most out of XML and Web services in PHP, this book is really grouped into three sections. The first section contains terminology and technical information about XML. This includes the concepts and structure of an XML document, validation, and other XML technologies commonly used. The chapters covering this information are based on various specifications. These specifications often use cryptic language and are difficult to understand, so I distill the information in clear terms.

The next group of chapters covers how to parse and manipulate XML documents using some of the extensions in PHP. I explain each extension and its API in detail with real-world examples to help reenforce the concepts covered. I also compare and contrast the extensions, providing you with some insight about where a particular extension excels and how it may not be the correct one to use in a particular situation.

The last group of chapters covers Web services. Although only a single native Web service extension exists in PHP (SOAP), I will provide in-depth coverage of additional technologies using the extensions from earlier chapters. In addition, I will cover how to integrate with the Yahoo, Google, Amazon, and eBay Web services.

Specifically, the chapters break down as follows:

Chapter 1, "Introduction to XML and Web Services": This chapter provides some background information about XML and Web services. In addition, the chapter defines what these terms mean, explains the history of how they came about, and shows some examples of how XML is used in the real world.

Chapter 2, "XML Structure": The XML 1.0 specification defines what XML is and the structure of documents but uses language that is not always so straightforward. This chapter explains the structure of an XML document in simple terms and provides some lucid examples. In addition, this chapter introduces some terminology used throughout the book.

Chapter 3, "Validation": This chapter explains the use of validation in XML using Document Type Definitions (DTDs), XML Schemas, and RELAX NG.

Chapter 4, "XPath, XPointer, XInclude, and the Future": The focus of this chapter is explaining how to write XPath expressions to query an XML document. You can use XPath with a few of the PHP extensions, and XPath serves as the foundation for XSLT in Chapter 10. The chapter also explains both XPointer and XInclude, which allow for more advanced XML processing.

Chapter 5, "PHP and XML": This chapter introduces the new XML support in PHP 5. It explains much of the functionality shared by the XML-based extensions, such as parser options, error handling, PHP streams, and document encoding.

Chapter 6, "Document Object Model (DOM)": This chapter provides an in-depth look at using the DOM extension and shows how it is used to manipulate an XML document.

Chapter 7, "SimpleXML": The SimpleXML extension provides a simple interface for working with XML documents. This chapter explains how to use the extension to access virtually any type of XML document, including more complex ones that use namespaces. 
Chapter 8, "Simple API for XML (SAX)": This chapter explains how to work with the xml extension and covers issues you may encounter when migrating an application that uses this extension from PHP 4 to PHP 5.

Chapter 9, "XMLReader": The XMLReader extension is a lightweight parser and an alternative to the xml extension covered in Chapter 8 . This chapter explains and demonstrates how to process an XML document using this extension.

Chapter 10, "Extensible Stylesheet Language Transformation (XSLT)": You can transform XML documents using XSLT. This chapter begins by explaining the XSLT specification in easy-to-understand terms. Then, this chapter shows how to use the XSL extension in PHP to perform transformations.

Chapter 11, "Effective and Efficient Processing": With a number of different extensions that can be used to work with XML in PHP, it is often difficult to decide which one to use. This chapter explains the differences between the extensions and continues with tips and tricks that can be used to optimally work with XML in PHP.

Chapter 12, "XML Security": Data integrity and data security are topics that every developer must be concerned with when writing applications. In this chapter, you will learn how to work with digital signatures and encryption as they pertain to XML.

Chapter 13, "PEAR and XML": The PHP Extension and Application Repository (PEAR) is a collection of software that can be used when writing an application. This chapter introduces PEAR and explores some of the XML packages it provides.

Chapter 14, “Content Syndication: RSS and Atom": Content syndication has become popular with the explosion of weblogs (blogs). This chapter examines the three formats that are used to syndicate data and shows how to create and consume syndicated feeds using the PHP extensions.

Chapter 15, "Web Distributed Data Exchange (WDDX)": This chapter explains what WDDX is and how you can use the wddx extension to exchange data between systems.

Chapter 16, "XML-RPC": This chapter examines the structure and exchange of XML-RPC documents. You will then learn about the xmlrpc extension and how you can use it to communicate with remote systems.

Chapter 17, "Representational State Transfer (REST)": Representational State Transfer (REST) is a simple method to create and consume Web services. I demonstrate how to create and consume REST-based services. In particular, you will see how to consume some real services from both Yahoo and Amazon.

Chapter 18, "SOAP": SOAP allows for the creation of complex Web services. The specifications involved are also quite complex. In this chapter, I show examples of both the Web Services Description Language (WSDL) specification and the SOAP specification. Using this knowledge, you will see how to use the SOAP extension in PHP using realworld examples from eBay and Google.

Chapter 19, "Universal Description, Discovery, and Integration (UDDI)": UDDI is a technology meant to make working with Web services easier. This chapter shows how you can use PHP to access and maintain records in a UDDI registry. 
Chapter 20, "PEAR and Web Services": Chapter 13 introduces PEAR and its XML packages; this chapter introduces you to some packages that you can use to create and consume a variety of Web services.

Chapter 21, "Other XML Technologies and Extensions": There are too many XML-based technologies to cover in a single book. In this chapter, I will introduce you to the XMLWriter and SDO XML Data Access Service extensions as well as show how to work with Ajax and Wireless Application Protocol (WAP) using PHP.

\section{Prerequisites}

Although the general information about XML and the different specifications pertain to any version of PHP, the tools and extensions covered in this book require PHP 5 or higher. For the greatest functionality, it is highly suggested that you use PHP 5.1 or higher because of the many enhancements and additional functionality in this release.

\section{Downloading the Code}

All the code featured in this book is available for download at the book's Web page, which you can find in the Source Code section at http://www. apress.com.

\section{Contacting the Authors}

You can contact the author at rrichards@php.net. 